\title{
ETUDES SUR LA TOXICITÉ DES HELMINTHES \\ I. LEUR TENEUR EN HISTAMINE
}

\author{
Par R. PAUtrizel, Mlle A. BEZIAN et J. BAILANGer
}

Les helminthiases humaines s'accompagnent de manifestations pathologiques dont la cause exacte est très mal définie. De nombreux auteurs l'expliquent par la présence dans les helminthes de substances qu'ils ont essayé d'isoler et qui se révèlent toxiques pour les animaux de laboratoire, avec des affinités pour tel ou tel tissu, variable suivant les travaux auxquels on se réfère.

Machebœuf et Mandoul (1939), puis Deschiens (1942, 1948), Deschiens et Poirier $(1947,1948)$ ont isolé de l'Ascaris megalocephala Cloquet 1824, par une extraction trichloracétique, une "substance toxique » dont l'injection au Cobaye est suivie d'une mort rapide, accompagnée de symptômes identiques à ceux du choc histaminique. Ces mêmes auteurs appliquèrent leur technique à d'autres helminthes du groupe des Cestodes et de celui des Trématodes, mais ne parvinrent à isoler aucun complexe dont la toxicité pour le Cobaye soit comparable à celui extrait de l'Ascaris megalocephala. D'après Deschiens et Poirier (1948), ces extraits trichloracétiques de Cestodes et de Trématodes peuvent cependant déterminer des intoxications chroniques chez les animaux auxquels on les injecte pendant une durée de 20 jours environ. Les lésions résultant de ces intoxications lentes rappellent de très près les lésions anaphylactiques.

Cependant, des intoxications aiguës ont été obtenues par Simonin (1921) après injection au lapin d'extraits de Tænia saginata Gœze 1782.

Il faut enfin signaler que les animaux sont préservés contre toutes ces manifestations aiguës par injection préalable d'antihistaminiques de synthèse.

Ces constatations avaient amené Deschiens et Bovet à rechercher l'histamine dans les extraits utilisés et également dans le liquide cælomique de l'Ascaris megalocephala. Leur conclusion fut la suivante: l'épreuve de recherche biologique de l'histamine est néga-

Ann, de Parasitologie, T, XXIV, $\mathrm{N}^{n s}$ 5-6. -1949 , p. 460-463. 
tive, et si l'histamine existe dans les produits essayés, c'est à des doses minimes non caractérisables $\left(0,1 \gamma\right.$ pour $\left.5 \mathrm{~cm}^{3}\right)$ (Deschiens, 1948).

Tout récemment, Cavier (1948) met en évidence, dans le liquide cælomique d'Ascaris suum Gœze 1782, des quantités d'histamine qu'il évalue de 200 à $250 \%$ pour $100 \mathrm{~cm}^{3}$, après dosage sur un fragment d'iléon de Cobaye suspendu dans du liquide de Tyrode sans glucose, oxygéné et à $38^{\circ} \mathrm{C}$. Il compare les contractions provoquées par l'addition d'une quantité définie de liquide cælomique fraîchement prélevé et celles obtenues en utilisant des quantités déterminées de chlorhydrate d'histamine pur. Il en conclut que les phénomènes toxiques décrits auparavant doivent être attribués en partie à l'histamine.

Nous avons cherché à le vérifier en reprenant systématiquement la recherche et le dosage de l'histamine dans les Ascaris suum, A. megalocephala et $A$. vitulorum Gœze 1782, et les liquides cælomiques correspondants. Nous avons également étendu nos recherches à quelques Cestodes (liquide hydatique de la larve d'Echinococcus granulosus Batsch 1786, strobile de Moniezia expansa Rudolphi 1810) et à un Trématode, Fasciola hepatica Linné 1758.

Recherche et dosage de l'histamine. - Il nous a paru nécessaire, en vue d'un dosage aussi rigoureux que possible, de procéder d'abord à l'extraction de l'histamine par un procédé chimique approprié. Nous avons appliqué pour cela, soit aux helminthes, soit à leurs produits parasitaires (liquide hydatique, liquide cælomique), la méthode de Barsum et Gaddum modifiée par Code (1937).

Les parasites apportés vivants de l'abattoir sont sur-le-champ lavés soigneusement à l'eau physiologique, essorés, et portés à $-25^{\circ}$ seulement les quelques instants nécessaires pour les amener à un état de congélation qui permette leur pulvérisation aussi parfaite que rapide. Le broyat d'helminthe est mis ensuite immédiatement au contact d'une solution d'acide trichloracétique pendant 30 à 60 minutes pour en éliminer les protéines. Dans un second temps, la totalité de filtrat obtenu est mise pendant 90 minutes en ébullition constante, au cours de laquelle il y a destruction de substances telles que l'adrénaline, l'acétylcholine, l'acide adénosinephosphorique, capables de perturber par la suite le dosage biologique de l'histamine. Le liquide ainsi obtenu est débarrassé de son acidité par distillation au bain-marie et sous pression réduite en présence d'alcool à $90^{\circ}$. L'extrait sec restant est repris par une quantité définie d'eau bidistillée.

Sur cette solution, nous pratiquons le dosage biologique de l'his- 
tamine selon la méthode classique de Guggenheim et Loeffler, utilisée par Code.

Remarque. - Le dosage n'est pratiqué que sur des portions d'iléon de Cobaye dont la parfaite sensibilité est soigneusement vérifiée auparavant, et qui donnent une contraction appréciable à 1/100 de $\gamma$ d'histamine.

La solution à doser est neutralisée au moment même du dosage.

Nous consignons dans le tableau suivant les résultats de nos dosages.

Les quantités d'histamine sont chacune évaluées en bichlorhydrate d'histamine pour un poids de 100 grammes du parasite ou de l'extrait parasitaire considéré :

Ascaris megalocephala $\delta$ et $q \ldots \ldots \ldots \ldots \ldots \ldots \ldots \ldots \ldots \ldots \ldots \ldots \ldots \ldots \ldots \ldots, 3 \gamma$

A. megalocephala $q$ (liquide cælomique) $\ldots \ldots \ldots \ldots \ldots \ldots \ldots, 8 \ldots \ldots \ldots \ldots$

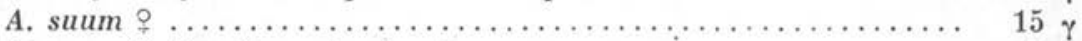

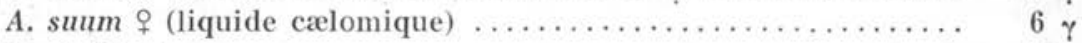

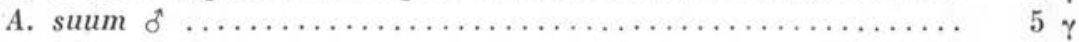

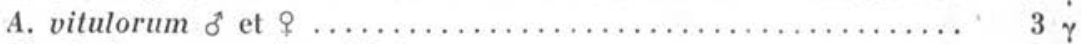

Moniezia expansa (strobile) .............................. $2 \gamma$

Echïnococcus granulosus (liquide hydatique) ................ ${ }^{6} \gamma$

Fasciola hepatica .................................. $29 \%$

Discussion. - Nous enregistrons pour les différents Ascaris des doses faibles et d'un même ordre de grandeur (3 à $15 \gamma$ ). L'espèce ou le sexe de l'helminthe considérẻ n'entraîne aucune variation d'un rapport constant ou qui soit susceptible d'intérêt. Peut-être faudrait-il considérer plutôt l'âge ou l'activité génitale du parasite.

L'absence d'histamine notée par Deschiens dans Ascaris megalocephala pourrait être due à une destruction spontanée de celle-ci, avant le dosage, ou à un manque de sensibilité de l'appareil ou du fragment d'intestin utilisé.

Au contraire, les fortes quantités rapportées par Cavier dans le liquide cælomique d'Ascaris suum prouveraient que ce liquide peut renfermer des substances étrangères à l'histamine, qui faussent la réaction en lui faisant perdre sa spécificité, d'où la nécessité d'une extraction préalable de l'histamịne.

Quant aux complexes toxiques isolés par certains auteurs, la substance de Machebœuf et Mandoul (1939) est exempte d'histamine éliminée au cours de la dialyse qui entre dans sa préparation. Il en est de même de l' "askaron » de Shimamura et Fujii (1917), car cet extrait est préparé par dessiccation, ce qui favorise l'action destructrice enzymatique de l'histaminase sur l'histamine. 
Mandoul (1939) et Deschiens (1942) n'obtiennent pas d'intoxication aiguë par injection aux animaux d'extraits de Cestodes ou de Trématodes. Cependant, ces parasites renferment des quantités d'histamine de même ordre de grandeur que celles contenues dans les Ascaris.

Il est à noter enfin que, chez l'homme, la teneur en histamine du sang normal est de l'ordre de 3 à $6 \gamma$ pour 100, et que celle des tissus (foie, poumons...) est d'un taux très supérieur. L'histamine apportée par les helminthes ne constitue donc qu'un bien faible apport pour provoquer des manifestations aiguës du type histaminique.

Nous pensons plutôt que, même à ces doses minimes, son influence se manifesterait par des modifications vasculo-conjonctives, lui donnant un rôle de facteur de diffusion des substances parasitaires.

\section{RÉsumé}

Après une extraction chimique appropriée et par des dosages błologiques, nous avons déterminé systématiquement la teneur en histamine de quelques helminthes. Les quantités évaluées sont trop faibles pour être rendues directement responsables des manifestations toxiques observées chez les porteurs de ces parasites.

\section{Bibliographie}

Cavier (R.). - C.R. Soc. Biol., CXLII, 1948, p. 898.

CODE. - J. of phys., LXXXIX, 1937, p. 257.

Deschiens (R.). - Bull. Soc. Path. exot., XXXV, 1942, p. 115.

Ann. Inst. Past., LXXV, 1948, p. 397.

Deschiens (R.) et Poirier (M.). - C.R. Acad. d. Sc., CCXXIV, 1947, p. 689.

- C.R Soc. Biol., CXLI, 1947, p. 445,988 et 1161.

- C.R. Soc. Biol., CXLII, 1948, p. 435.

Macherceuf (M.) et Mandoul (R.). - C.R. Soc. Biol., CXXX, 1939, p. 1032.

- C.R. Soc. Biol., CXXXII, 1939, p. 124.

Mandout (R.). - C.K. Soc. Biol., CXXX, 1939, p. 1035.

C.R. Soc. Biol., CXXXII, 1939, p. 126.

Shimamura (T.) el Fuji (H.). - J. Coll. agric., Tokyo, III, 1917, 189.

Simonin (P.). - Thèse Médecine Nancy, 1921.

Laboraloire de parasitologie de la Faculté de médecine de Bordeaux. 